www.jmscr.igmpublication.org

Impact Factor (SJIF): 6.379

Index Copernicus Value: 71.58

ISSN (e)-2347-176x ISSN (p) 2455-0450

crossrefDOI: https://dx.doi.org/10.18535/jmscr/v6i6.188

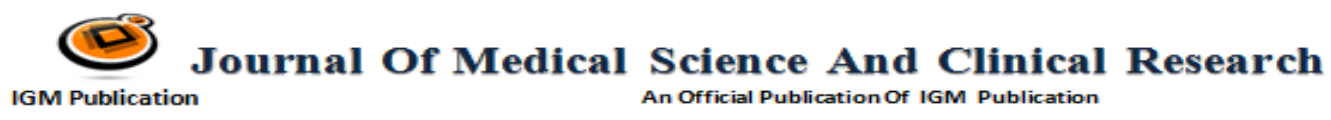

An Official Publication of IGM Publication

\title{
The Assertiveness Training Program on the Coping Skills of the Abused Female Adolescents
}

\author{
Authors \\ Sister Gracia T. Gegajo, DSFS (PhD Cand.), Marieann Vargas, PhD Psychology \\ University of Santo Tomas, Espana Manila, Philippines
}

\begin{abstract}
This study aims to determine the effect of the Assertiveness Training Program on the Coping Skills of the Abused Female Adolescents in Marillac Hills Crisis Center for Children in Alabang, Muntinlupa City, Philippines. Thirty abused female adolescents randomly assigned to the experimental and control group. The design used in this study was an experimental design of Pretest Posttest control group design that allowed a comparison between the experimental and control group in terms of their pretest and posttest scores as measured by the A-COPE (Adolescent Coping Orientation for Problem Experiences). The Assertiveness Training Program (ATP) devised by the author (of eleven modules in twenty-two sessions) was applied to the experimental group for one and a half month.

Findings revealed that a significant difference was found between the pretest and posttest of the experimental group signifying that Assertiveness Training Program was effective in causing a change in the Coping Skills of Abused Female Adolescents.

Keywords: Assertiveness Training Program, Coping Skills, Abused Female Adolescents.
\end{abstract}

\section{Background of the Study}

Abuse rooted in one's childhood can lead a person to psychological problems like depression, low self-esteem and unassertiveness. Symptoms of disturbance can be manifested in one way or another leading the person to an ego dysfunction. What possible intervention then can be done in order to help them develop necessary social skills and improve their coping skills so that they can manage their interpersonal relationships and situations effectively and make themselves feel better? In addressing this issue, all of us are confronted with a big responsibility because we are in contact and related in one way or another with these adolescents.

Pope John Paul's message to young people in his visit here in the Philippines during World Youth
Day in 1995 stated and urged these to the adolescents: "Young People, You are The Promise of Tomorrow" and he continued saying, "Be bearers and transmitters of Joy! It is only when you have joy and peace that you will construct something worthwhile for men and women. Be real builders of society.' (A Collection of Pope John Paul II's messages to the youth). But they can only be builders of society when their personal being and individuality are well and integrated and that does not make them worry of their self-image. In short, that makes them assertive by building up their coping skills. Called by this concern to respect and renew life, the researcher was motivated to think and create a way to be able to share a concrete instrument to be of use to improve the life of these abused 
adolescents in a humane world wherein they can be at peace with the society they live in and to be able to face life in themselves with their skills to be assertive and that is to hone and improve their coping skills as their plus factor in their challenges in life.

\section{Conceptual Framework}

Figure 1 shows the schematic diagram of the assertiveness training program on coping skills of the abused adolescents

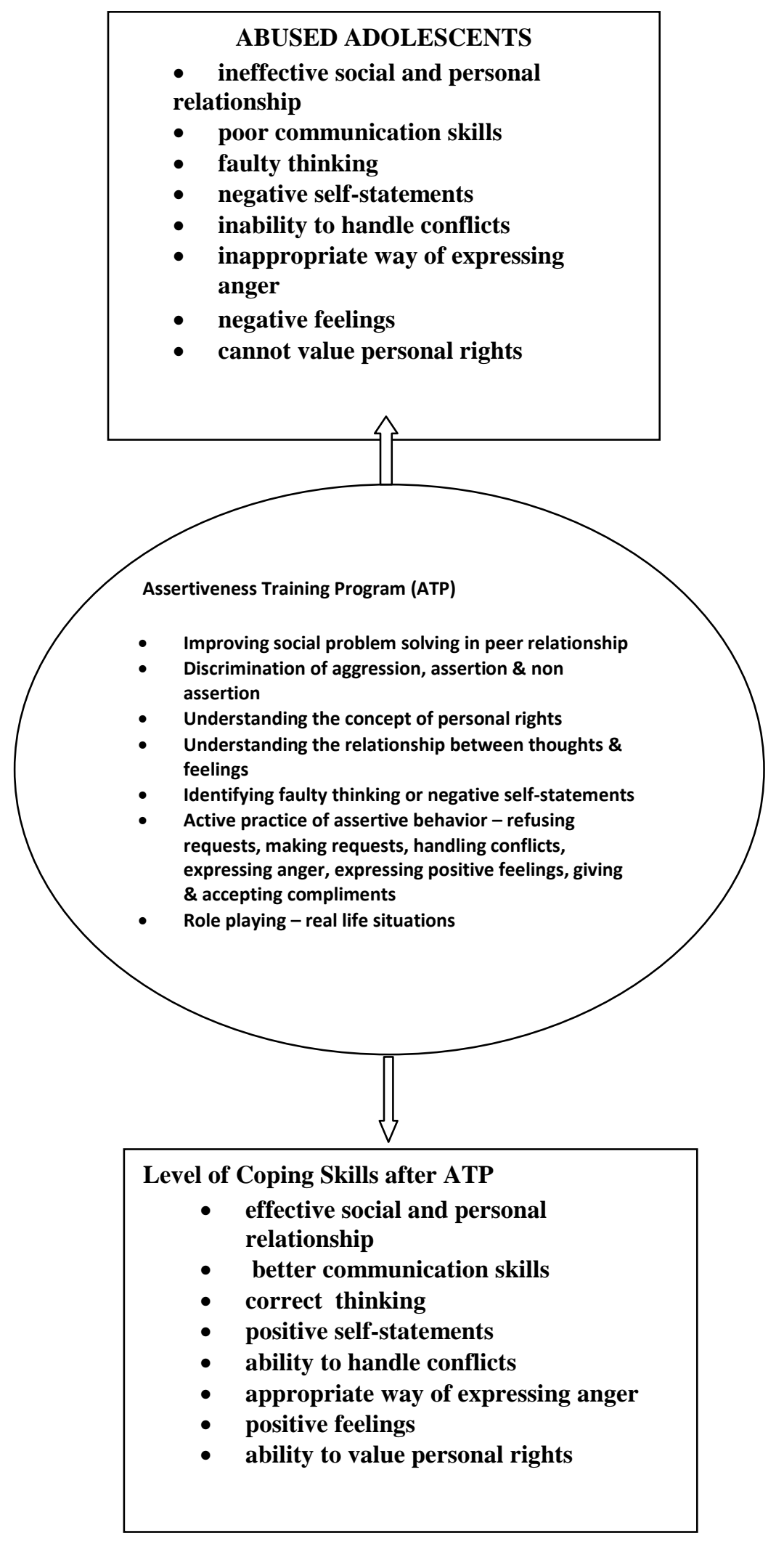


The figure showed that abused adolescents have manifestations of ineffective personal and social relationships, poor communication skills, faulty thinking, have negative self-statements, cannot handle conflicts well, have inappropriate way to express anger, have negative feelings, and cannot value personal rights; and when subjected to the Assertiveness Training Program which is about on improving social problem solving in peer relationship, discrimination of aggression, assertion and non-assertion, understanding the concept of personal rights, understanding the relationship between thoughts and feelings, identifying one's faulty thinking, active practice of assertive behavior and role playing on real life situations could possibly increase the level of their coping skills and effective social and personal relationship, better communication skills, correct thinking, positive self-statements, ability to handle conflicts, appropriate way of expressing anger, positive feelings and ability to value personal rights that could be evident in them.

The conceptual framework that guided this study was based on the behavioral procedure for teaching individuals on how to express their opinions and rights without abusing the rights of others and that is assertiveness training. It uses modeling to improve deficient social or interpersonal skills. Assertiveness training program for the abused adolescents is very important since it is a behavioral technique that can help them develop the necessary social skills to manage effectively interpersonal situations and for them to feel better of themselves in those interpersonal situations. This requires them a change of behavior and attitude, learn how to judge when it is appropriate and reasonably stand on their ground than giving in to others. It teaches the person to assert one's own right in a nonaggressive manner and to learn how to deal with others to get what he/she intends to get in relationship with them. It is enabling the persons to act in their own best interest by expressing honestly and directly their thoughts and feelings. The researcher therefore came up with an idea to establish an assertiveness training program to help these abused adolescents in their coping skills to find out if this could be a useful method in enhancing one's social skills and interpersonal relationship.

\section{Literature Review}

\section{Assertiveness}

Assertiveness is a form of behavior and not a personality trait. Because it is a form of behavior, it is learnt, and can be learnt, no matter how helpful or unhelpful people's existing habit patterns may be to the process of learning. (Willis \& Daisley, 1995)

It is the ability to express feelings and thoughts in a socially appropriate manner. (Fensterheim, 1975). It involves standing up for one's personal rights and expressing thoughts, feelings, and beliefs in direct, honest and appropriate ways which do not violate another person's rights. (Lange \& Jakubowski, 1976) It is standing for one's own right without infringing upon the rights of others; an adaptive behavior because it is functional in a given context, is self-enhancing, generates positive feelings toward oneself and others, and leads to smooth interpersonal relationship. (Dawley,1995)

Assertiveness is a core interpersonal behavior and is key to human relations. It has many forms. According to Alberti and Emmons (1992) assertiveness is viewed as a skill and capacity of interpersonal communication. (Yen, et.al, 2004) It is the ability to express your feelings, to choose how you will act, to speak up for your rights when it is appropriate, to enhance one's self-esteem, to help oneself develop self-confidence, to disagree when you think it is important, and to carry out plans for modifying your own behavior and asking others to change their offensive behavior. (Bower, 1976)

\section{Assertiveness Training Program}

An Assertiveness Training Program is designed to improve an individual's assertive beliefs and behaviors which can help the individual change, how they view themselves, establish self- 
confidence and interpersonal communication. (Weston \& Went, 1999, Alberti \& Emmons, 2001 \& Lardizabal 2001)

In several studies that have explored the influence of an assertiveness training program on assertiveness, subjects include alcoholics, university students with low assertiveness, nursing staff, in-patients and out-patients from the department of psychiatry (Pfost et.al., 1992, Lee \& Crocket, 1994, Chen 1995 \& Weinhard et. al., 1998). Six to eleven training sessions with one to two hours duration were given. The Rathus Assertiveness Schedule, Assertive Scale, and Behavioral Assertiveness Test- Revised were used. Past studies indicated that assertiveness of subjects was significantly increased after training; follow-up measurements taken four weeks to four months after the end of the training were all significantly increased. Additionally, Meyer (1991) found that fewer than six training sessions did not have a significant effect on improving assertiveness.

\section{Coping}

Coping refers to a person's cognitive and behavioral responses to a stressful situation. It includes what we do and think in response to a stressor, even if we are unaware of why or what we are doing. (DeLongis, A. \& Newth, S. 2001) It is referred to a person's general approach in dealing with stress as his or her coping style". (Burger, 2000)

Coping is an essential part of affirmation, but not synonymous with affirmation. The word affirmation comes from the Latin affirm are and it means to make firm, to give strength to, to make strong.

Some coping patterns like positive reinterpretation, active coping and planning are associated with low anxiety and high self-esteem. Other coping strategies are associated with lower self-esteem and anxiety. Some coping strategies appear to produce greater adaptive value compared to others; no coping strategy can ensure a successful outcome. This is because the adaptive value of coping strategy depends on the exact nature of the situation. Hence, in some instances, even ill-advised coping strategies may have adaptive value. (Santiago 2004)

Recent studies that have investigated creativity, self-actualization and coping skills found that selfactualizing creative individuals seem to have effective coping skills. (Runco et al., 1991 \& Sheldon, 1995 in Whurr, 2005).

\section{Types of Constructive Coping or Healthy Coping Skills}

1.Appraisal Focused Coping- is facilitated by rational emotive therapy which involves alteration of client's pattern of irrational thinking to reduce maladaptive emotions and behavior. It is facilitated by Ellis' suggestions on how to reduce catastrophic thinking by digging out the irrational assumptions that cause it. This also includes strategies using humor in dealing with stress and looking for the positive aspect of setbacks and problems. (Santiago, 2004)

2. Problem-Focused Coping - this includes those forms of coping that geared directly toward solving the problem or changing the stressful situation. (Briones, 2007). A four- way process can be facilitated under this; first, clarifying the problem; second, generating alternative courses of action; third, evaluating one's alternative and selecting a course of action and fourth, taking action while maintaining flexibility. The skills that can be used here are: improving self-control, seeking help and time management or effective use of time.

3. Emotion- Focused Coping - these are forms of coping that are geared toward managing one's emotions during stressful situations. It includes distracting oneself, releasing pent-up emotions, relaxation exercises and meditation. Distraction involves diversion of one's attention from the problem by thinking other things or engaging in other activities requiring attention. Releasing pent-up emotions is psychologically beneficial since it helps the person to avoid physiological problems like increased blood pressure. 
Abused adolescents, just like adults must have coping strategies in dealing with their daily stress in life. With this, Formaran in 1993, emphasized that since coping strategies are learned, it can be taught. She said that schools provide an appropriate setting wherein coping skills can be taught by classroom teachers who had specific trainings or by other service professionals. She believed that coping skills is related to the basic mission of the school which is to prepare the youth to effectively function in a society.

\section{Methodology \\ Research Design}

The design used in this study was an experimental design. This experimental design allowed a comparison between the experimental and control group in terms of their pretest and posttest scores as measured by A-COPE (Adolescent Coping Orientation for Problem Experiences).

\section{Research Participants}

The participants who were purposively selected for this study were comprised of thirty abused female adolescents who were presently housed in Marillac Hills Crisis Center for Children in Alabang, Muntinlupa City. They were randomly assigned to the experimental and control group. Fifteen were assigned to the experimental group and the other fifteen to the control group. The experimental group was subjected to assertiveness training program sessions, while the control group was given a supervised study period wherein the abused adolescents do their usual routine assignments and supplemental activities that were assigned to them. The assertiveness training program was not discussed to the subjects of the control group.

The method used was a Purposive Sampling Technique. The selection criteria were the following: (1.) abused female adolescents with ages of 11 to 17 year old (2.) is sheltered in the Marillac Hills Crisis Center for Children; (3) is abused in any form physically, emotionally, sexually and psychologically.(4) is willing to undergo assertiveness training program sessions; (5) has low score in A-COPE.

\section{Research Instruments}

The researcher used the following instruments: the Assertiveness Training Program Modules and the A-COPE Adolescent Coping Orientation for Problem Experiences.

1.) The Assertiveness Training Program or ATP - an instrument constructed and devised by the researcher. Made up of twenty two sessions (eleven modules), translated into Filipino language by a Filipino Subject College Professor of San Beda College of Sampaloc Manila, Philippines and double checked by the author herself and by another professor.

It contains behavioral techniques to help the abused female adolescents with the necessary social skills in managing interpersonal relationships and situations better requiring a change of behavior.

Sessions were about on improving social problem solving in peer relationship; discrimination of aggression, assertion and non-assertion; understanding concept of personal rights; understanding relationship between thoughts and feelings; identifying faulty thinking; active practice of assertive behavior on refusing requests, making requests, handling conflicts, expressing anger, expressing positive feelings, giving and accepting compliments and role playing on real life situations. These were given for one and a half month, meeting them every Monday, Wednesday and Friday from 10:00 am to 12:00 noon in one to two sessions per meeting.

A dry-run was done by the author to the adolescents of the depressed areas of Sitio Puloand Barangay Medina of Talon IV, Las Pinas City, Philippines every Sunday afternoon from 3 to $6: 30 \mathrm{pm}$ to facilitate the session better on the day of the implementation of the ATP.

2.) The A-COPE - Adolescent Coping Orientation for Problem Experiences. 
The A-COPE is a fifty four-item questionnaire developed by Joan $M$. Patterson and Hamilton McCubbin in (1991) designed to measure self- reported coping behaviors and strategies in adolescents. The instrument measures coping patterns along these subscales (i.e., developing self-reliance and optimism; developing social support; solving family problems; seeking spiritual support; investing in close friends, engaging in demanding activity).

An important characteristic of the A-COPE inventory is that the instrument is based on theory that integrates individual coping theory and family stress theory. It is scored by summing item scores for a total score; several items are reverse scored. The total score of the A-COPE can be used as overall measure of coping.

The same college professor of the Filipino Subject of San Beda College, Mendiola, Philippines translated the A-COPE and after translating it in Filipino language the researcher asked the help of an English Major teacher and former Principal of the Don Carlo Cavina High School (the school where the author is rendering her mission as a guidance counselor to the high school students) to have it translated back to English. This was done to find out that the instrument was easy to understand. These were pre-tested to thirty seven female adolescents of Sitio Pulo and Barangay Medina of Talon IV of Las Pinas City., Philippines which were randomly selected.

\section{Treatment Procedures in the Conduction of the Experiment}

Pre-test was given to all the qualified abused adolescents as a baseline for the study and only thirty of them who got a low score on the coping skills were taken as participants for the experimental group and control group, randomly assigning them with the use of a fishbowl technique. After which, the Assertiveness Training Program sessions were given to the fifteen abused female adolescents who belong to the experimental group (Group A) for twenty two sessions, thrice a week (Monday, Wednesday and Friday) for a period of one and a half month with one to two sessions every meeting to enhance the coping skills of the abused female adolescents while the control group had a supervised study period wherein they made their usual assignments and supplemental activities that was assigned to them. After the Assertiveness Training Program Sessions, post-test was given to both the control and experimental groups in order to find out its effects on the Coping Skills of the Abused Female Adolescents.

\section{A. Pre-Experimental Stage Selection of Participants}

A letter was sent to the executive director of DSWD National Capital Region (NCR) in Sampaloc, Manila, Philippines to secure permission to conduct the experiment on Assertiveness Training Program in Marillac Hills Crisis Center for Children in Alabang, Muntinlupa City since it was under this Department. A copy of the letter was given to the head of the staff of Marillac Hills Crisis Center for Children in Alabang, Muntinlupa City by the NCR through fax machine to formally inform them of the assertiveness training program that the author intends to do and to provide the researcher the respondents that will undergo this program.

The researcher visited the shelter for some days prior to the experiment to meet the psychologists for the schedule of the prospective participants, and to establish rapport with them. The A-COPE was administered to the selected female adolescents to get the baseline of the participants and only thirty abused female adolescents who obtained a low score were selected for the study as experimental (group A) and control group (group B). Those who were selected to participate were informed that they will be randomly assigned into two groups and will be separated from their peer groups or friends. The researcher explained that the reason in doing this is for them to get a chance to know also their other companions in the shelter. 
The Personal Data Form was given and completed; their signature was affixed as a sign of their consent to join the study.

After which, the participants were randomly assigned into two treatment conditions using the fish bowl technique. The names of the participants were placed in a box, mixed together and then the researcher drew their names one by one at random. The first half (15 of them) was included in Group A (Experimental Group) and the other half (another 15 of them) was assigned in Group B (Control Group). Both the experimental and control group had an equal number of participants, that is, fifteen per group.

Participants in both the experimental and control groups were not informed that they will undergo an experimental study. Instead, those who were in group A were informed that they will attend a Training Program with the researcher as their speaker and group B will have another program.

\section{The Setting}

The experimental study was conducted in the Marillac Hills Crisis Center for Children in Alabang, Muntinlupa City, Philippines.

\section{B. Experimental Stage}

\section{Assertiveness Training Program Sessions}

Assertiveness Training Program Sessions was done to the experimental group (Group A) and was carried out for one and a half month. One to two sessions in one half day in the morning and thrice a week was given to them. Thus, the twenty two sessions that was given to the experimental group were in modular forms. The session started from 10:00 am until 12:00 noon.

Upon completion of the twenty two sessions on Assertiveness Training Program for one and a half month, the researcher administered the Post-test of A-COPE to the experimental group of the abused adolescents. Their scores were recorded, statistically analyzed and interpreted.

A Post-test A-COPE was also given to the participants of the control group to find out the scores of their coping skills.
Modules of ATP (Assertiveness Training Program) that will be the coping skills instrument for the participants:

Module 1: Is entitled "Getting to know You and Small Talk". This is session 1 and 2 with the activities objectives of communicating oneself through the experience of self-disclosure to their companions. They have to communicate and be socially involved and have the chance to practice their skills in making small tasks.

Module 2: This is session 2 and 3 entitled "The Contract" and "Purpose of Being Here". This is about having an opportunity to practice Assertiveness skills by expressing their needs, opinions in a constructive way.

Module 3: This is about better understanding the concept of assertiveness, passiveness and aggressiveness (session 5 and 6). Choosing one's vocabulary and offering situations in real life can help change interpretation of meaning.

Module 4: This is about identifying areas of their lives where they can practice and demonstrate their assertion skills. Appraisal of their present behavior pattern is highlighted. (Session 7 and 8).

Module 5: Discussing a nonassertive behavior as a negative sign for one's well-being. Identifying and acknowledging positive personal characteristics as a plus vibes. (Session 9 and 10). Module 6: This is session 11 and 12. Recognizing assertiveness as a choice of behavior, choosing appropriate behavior to meet difficult situations in life. Looking at ways to overcome barriers to assertiveness.

Module 7: Evaluating Assertiveness skills as leaders and mirroring body language in the development of interpersonal relationship. This is sessions 13 and 14.

Module 8: This is about being aware of other's expectations that directs out one's own actions and identifying areas of irrational beliefs as barrier to assertive behavior. Sessions 15 and 16 .

Module 9: This is session 17 1nd 18. Refusing requests in an assertive way and role playing. 
Module 10: Handling conflicts and enhancing interpersonal skills and self-worth. Sessions 19 and 20.

C. Post- Experimental Stage - a session on feed backing regarding the Assertiveness Training Program was done to find out if the said program had an effect on the abused adolescents individual and group social and coping skills. This was done through interview and their narration of their personal and group experience.

\section{Results and Discussion}

\section{Presentation of Data, Analysis and Interpretation}

Table 1 Pretest and Posttest Coping Skills Mean Scores and Standard Deviation Values of the Participants when grouped according to Experimental and Control.

\begin{tabular}{|c|c|c|c|c|}
\hline \multirow{2}{*}{ Groups } & \multicolumn{2}{|c|}{ Pretest } & \multicolumn{2}{c|}{ Posttest } \\
\cline { 2 - 5 } & M (SD) & Int. & M (SD) & Int. \\
\hline \multirow{3}{*}{ Experimental } & 156.33 & Below & $\mathbf{1 7 1 . 6 7}$ & Average \\
\hline \multirow{3}{*}{ Control } & $(8.83)$ & Average & $(12.99)$ & \\
& 156.00 & Below & 158.07 & Below \\
& $(9.74)$ & Average & $(11.97)$ & Average \\
\hline
\end{tabular}

It can be gleaned from Table 1 the mean scores in the pretest and posttest as well as the standard deviation values of the two groups. The Mean measures the central tendency or position of scores. It may also refer to the average.

The A-COPE total score was used to measure the overall coping skills of the respondents. In the manual of the A-COPE, actual norms were not available, though there were some several samples of adolescents with a total mean of 168.7 (SD 26.3). In the local norm made by the researcher a mean of 172 and an SD of 17.89 was obtained.

By inspection, the table showed the difference in the coping skills mean scores of the participants in the pretest of the experimental group which is 156.33 and is Below Average and this is characterized by the manifestations of pessimism, unreliability, inability to develop social support, cannot solve family problems, inability to seek spiritual support, cannot invest in close friends and inability to engage in demanding activity; in the posttest coping skills mean scores of the experimental group however, a score of $\mathbf{1 7 1 . 6 7}$ was obtained which increases to $\mathbf{1 5 . 3 4}$, implying that there was an increase in the coping skills of the participants after the Assertiveness Training Program was given which makes it Average. On the other hand, for the control group the table shows not much difference in the coping skills mean scores of the participants in the pretest and posttest of the control group which is 156.00 in the pretest and 158.07 in the posttest which increases only by 2.07 and is Below Average.

In terms of the coping skills of the participants of the experimental and control groups since all the SD values were large, means that more or less the participants do not possess the same characteristics with regards to their coping skills, thus supporting of what Burger (2000) mentioned that "coping is referred to a person's general approach in dealing with stress as his or her coping style".

Butler.(1992) also stated that women in particular have often been taught to hide their feelings and preferences and try to get their way by manipulation or other indirect means and in additional to emotional and psychological benefits, takes a more active approach to selfdetermination showing to have positive outcomes in many personal choices related to health, including being assertive in risky sexual situations and assuming responsibility for self-care. Moreover, supporting the statement of Alberti and Emmons (2001) stating that Assertiveness Training enhances sense of well-being and more positive self-esteem which many participants report that they feel better about themselves and are more capable of handling the stresses of daily life. This form of training is tailored to the needs of specific participants and situations where in they find particularly challenging, a training that can be applied to much different personal, academic health care and work situations. 
Table 2 Significant Difference between the experimental and control group in the pretest and posttest condition.

\begin{tabular}{|c|c|c|c|c|}
\hline $\begin{array}{c}\text { Type of } \\
\text { Test }\end{array}$ & $\begin{array}{c}\mathbf{U} \\
\text { Value }\end{array}$ & $\begin{array}{c}\mathbf{p} \\
\text { Value }\end{array}$ & Interpretation & Decision \\
\hline Pretest & 104.55 & .991 & $\begin{array}{c}\text { Not } \\
\text { Significant }\end{array}$ & Accept \\
\hline Posttest & $\mathbf{4 8 . 0 0}$ & $\mathbf{. 0 1 2}$ & Significant & Reject \\
\hline
\end{tabular}

Note: * Significant if $p \leq 0.05$

It can be observed in Table 2 that for the pretest, no significant difference in the coping skills of the participants of the experimental and control group was found before the experiment. This is because the $\mathrm{p}$ (probability) value which is .991 is greater than 0.05. This is an indication that at the start of the experiment, the two groups have the same level of coping skills. However, for the posttest a significant difference in the coping skills of the participants of the experimental group and control group was found after the experiment. This is because the $p$ (probability) value which is $\mathbf{. 0 1 2}$ is less than 0.05. This is an indication that after the experiment was conducted, the groups now have different level of coping skills, thus rejecting the null hypothesis.

The result for the pretest further supports the statement of Sue et.al. in 2005 which states that "children who are abused are more prone to manifest significant direct and indirect physical and psychological problems. Psychological effects of abuse include depression, aggressive impulsivity, low self-esteem, self-destructive behavior and impaired memory. These abused children may show excessive anxiety, which can lead to eating and sleeping disorders, nightmares, bedwetting, and in the teenage years - conduct disorders.

Moreover, in the study of Myers, Templer and Brown in 1984 of the coping ability in women who become victims of rape, it proved that the strongest domain of prediction was psychosocial competency with the rape victim scoring lower on measures of social presence, dominance and assertiveness and higher on external/ social locus of control.

For the posttest, the foregoing results further supports previous research studies of Morgan and Leung in 1980 wherein they made a pilot study focused on the effects of Assertion Training on 14 physically disabled undergraduates' acceptance of disability. A pretest-posttest control group design was utilized and subjects were randomly assigned to either a treatment group or a no-treatment control group. The hypotheses analyzed were that subjects given Assertion Training would show increases in Acceptance of Disability Scale scores, self-concept/ esteem scores (Rathus Assertiveness Scale) and social interaction skills scores (Behavioral Observation Scale) when compared with individuals who did not experience Assertion Training. Significant Results were obtained for all hypotheses indicating that the groups differed in acceptance of disability, self-concept and social interaction. Thus, suggesting that Assertion Training may be effective for increasing acceptance of disability in physically disabled students.

In addition to this, Gallin in 1984, Baggs and Spence in 1990, Wise et.al. in 1991, Sharon in 1997 and Korsgard et.al., in 1998 proved that past studies have found that assertiveness training improved human relations, positive interpersonal behavior, and interpersonal reactions, and decreased social anxiety thus supporting the rejection of the null hypotheses.

Table 3 Significant Difference between the Pretest and Posttest Coping Skills Mean Scores of the participants of the Research Groups.

\begin{tabular}{|l|c|c|c|c|}
\hline \multicolumn{1}{|c|}{ Groupings } & $\begin{array}{c}\text { T } \\
\text { Value }\end{array}$ & $\begin{array}{c}\text { P } \\
\text { Value }\end{array}$ & Interpretation & Decision \\
\hline $\begin{array}{l}\text { Experimental } \\
\text { Pre and Post }\end{array}$ & $\mathbf{0}$ & $\mathbf{. 0 0 1}$ & Significant & Reject \\
\hline $\begin{array}{l}\text { Control Pre } \\
\text { and Post }\end{array}$ & 5 & .365 & $\begin{array}{c}\text { Not } \\
\text { Significant }\end{array}$ & Accept \\
\hline
\end{tabular}

Note: Significant if $\mathrm{p} \leq \mathbf{0 . 0 5}$

Table 3 showed that a significant difference was found between the pretest and posttest of the experimental group because the $\mathrm{p}$ (probability) value is less than 0.05 , which is $\mathbf{. 0 0 1}$ which means 
that the independent variable which is the assertiveness training program was effective in causing a change in the dependent variable which is the coping skills of the participants, rejecting therefore the null hypothesis. However, no significant difference was found between the pretest and posttest of the control group because the $\mathrm{p}$ (probability) value is greater than 0.05 , which is .365 and which mean that in the absence of the independent variable which is the assertiveness training program, no change occurred in the dependent variable which is the coping skills of the participants, thus, accepting the null hypothesis.

This supports the study of Stake and Pearlman in 1980 on assertiveness Training as an intervention technique for low performance self-esteem women wherein findings suggest that assertiveness Training is a viable technique for improving low performance self-esteem among women.

Moreover, Debra Buback's III RN study on Assertiveness Training to prevent verbal abuse in Operating Room (OR) that focuses on conflict resolution and communication skills supported that Assertiveness Training is an effective method of coping with verbal abuse, thus making it as a purpose to educate preoperative nurses about the effect of Verbal Abuse and how to implement an Assertiveness self-learning module to prevent future episodes of Verbal Abuse.

In addition to this, Glueckauf and Quitness also support the outcome of this study with their research in 1992 in Purdue University in Indianapolis, USA on a special population of thirty four physically disabled adults who had a trauma. The subjects participated in an 11- week Assertiveness Training Program and overall results support the use of Assertiveness Training in enhancing perceived social efficacy and interpersonal skill of physically disabled adults in wheelchairs.

Moreover, Brecklin in 2008 made a review on Evaluation Outcomes of self-defense training for women, a training that gives women access to a new set of assertive and combative responses to various forms of intimidation and threat along the continuum of sexual violence. The main goals of self-defense for women includes assertiveness. A study of 1623 sexual assaults victims showed that victims with pre-assault self-defense/ assertiveness training were marginally more likely to avoid completed rape than victims without training, implying that the training may have given these women the tools to prevent the escalation of their attacks. Further supported by the multivariate finding that victims with preassault training were more likely to say that their resistance stopped the offender or made him less aggressive than victims without training. In other words, training participants demonstrating that self-defense/ assertiveness training may help women to more successfully fend off attackers thus, supporting the study made by the researcher.

Table 4 Measure of Effect Size of the Assertiveness Training Program on the Coping

Skills of the Participants.

\begin{tabular}{|c|c|c|}
\hline Groupings & $\mathrm{r}$ & Interpretation \\
\hline $\begin{array}{c}\text { Experimental } \\
\text { Group }\end{array}$ & .64 & Medium effect \\
\hline
\end{tabular}

Table 4 showed the effect of Assertiveness Training Program on the Coping Skills of the participants. The effect size estimate which is $r=$ .64 is considered medium. Since in Cohen's guide for interpretation 0 to 0.2 has small effect, 0.3 to 0.8 has medium effect and 0.9 and up has a large effect. This signifies that there was a medium effect on the coping skills of the participants in the experimental group compared to the control group, thus, confirming that the Independent variable which is the Assertiveness Training Program was effective in causing a change in the dependent variable which is the Coping Skills of the participants. Further supporting Alberti and Emmons statement in 1992, mentioning that assertiveness is viewed as a skill and capacity of interpersonal communication and as stated by Yen et. al, 2004 it is a core interpersonal behavior and is key to human relations. An ability to express one's feelings as 
said by Bower, 1976; to choose how one will act, to speak up for one's rights when it is appropriate, to enhance one's self-esteem, helping oneself to develop self-confidence, to disagree when one thinks it is important, carrying out plans for modifying one's own behavior and asking others change their offensive behavior.

As mentioned by Weston and Went, 1999; Alberti and Emmons, 2001 and Lardizabal, 2001, an Assertiveness Training Program is designed to improve an individual's assertive beliefs and behaviors which can help the individual change, how they view themselves, establish selfconfidence and interpersonal communication.

With this Formaran in 1993, emphasized that since coping strategies are learned these can be taught. She suggested some intervention approaches in teaching children and adolescents coping skills in school settings. Interventions that are based on behavioral and cognitive- behavioral therapeutic procedures that include assertiveness training, self- instructions training, rationalemotive therapy, relaxation training, stress inoculation training, attribution retraining, behavioral self-management training, social problem solving training and social skills training.

\section{Summary of Findings and Conclusions}

Findings drawn from the study are as follows:

The pretest coping skills mean scores of the experimental group is 156.33 with an SD of 8.83 and the control group which is 156.00 and an SD of 9.74 are below average. The posttest coping skills mean scores of the control group is 158. 07 with an SD of 11.97 are also below average. However, the posttest coping skills mean scores of the experimental group is 171. 67 with an SD of 12.99 is average. This signifies that before the experiment both the experimental and control group pretest coping skills mean scores were below average and after the Assertiveness Training Program, the coping skills mean scores of the experimental group increased which became average while that of the control group remained below average.
No significant difference was found in the coping skills of the participants of the experimental and control group before the experiment which indicates that at the start of the experiment the two groups have the same level of coping skills.

A significant difference however in the coping skills of the participants of the experimental and control group was found after the experiment which indicates that after the experiment was conducted, the groups now have different coping skills.

A significant difference was found between the pretest and posttest of the experimental group which means that the Assertiveness Training Program was effective in causing a change in the coping skills of the participants.

No significant difference was found instead between the pretest and posttest of the

control group which means that in the absence of the Assertiveness Training Program no change occurred in the coping skills of the participants.

\section{Conclusion}

There was a medium effect on the coping skills of the participants in the experimental group compared with the control group. It can therefore be concluded from the findings of the study that the Assertiveness Training Program was effective in causing a change in the Coping Skills of the abused female adolescents.

\section{References}

\section{Books}

1. Alberti, R.E., Emmons M.L., Your Perfect Right: A Guide to Assertive Behavior $\left(20^{\text {th }}\right.$ Edition, Impact Press, San Luis Obispo, CA, 1992).

2. Alberti \& Emmons., Your Perfect Right. Assertiveness and Equality inYour Life and Relationships (18 ${ }^{\text {th }}$ Edition, Impact Publishers, Alascadero, LA, USA, 2001).

3. Bower, S. Anthony and Bower, Gordon H., Asserting Yourself, A Practical Guide for Positive Change (1976). 4.

4. Burger, J., Personality (Thomas Learning, Wadsworth CA, 2000). 147. 
5. Chen, S.R., 1995. The Effect of Assertion on Assertiveness and Personality Trait. Unpublished Thesis. University of National Taiwan Normal, Taipei.

6. Dawley, Harold Jr. H., Achieving Assertive Behavior (Brooks Cole Publishing Company Inc. 1995).

7. DeLongis, A. \& Newth, S., Assessment \& Therapy. Coping with Stress. Specialty Articles from the Encyclopedia of Mental Health.(Academic Press San Diego, CA., USA 2001).

8. Formaran, Susan G. Coping Skills Interventions for Children and Adolescents. (Jossey- Bass Incorporation San Francisco. 1993).

9. Fensterheim, H. \& Baer, Jr. Don't Say Yes When You Want to Say No. New York: David McKay Co. Inc. 1975.

10. Lange A., S. Kakubowski, P., Reponsible Assertive Behavior.Champaign III. Research Press, 1976.71

11. Sue, David et. al. Essentials of Understanding Abnormal Behavior. Houghton Mifflin Company. USA. 2005. 350-351.

12. Willis, Liz \&Daisley, Jenny.,The Assertive Trainer. (McGraw-Hill Book Company Europe 1995).

13. Whurr Publisher Limited., Promoting health through Creativity for Professionals. (PA, USA 2005). 9.

\section{Published/Unpublished Journals/Thesis}

1. Baggs K., Spence, S. H., 1990. Effectiveness of Booster Sessions in the Maintenance and Enhancement of Treatment Gains Following Assertion Training.Journal of Consulting and Clinical Psychology.Vol 58. No.6. 845854.

2. Brecklin, Leanne R., 2008. Evaluation Outcomes of Self-Defense Training for Women: A review. Aggression and Violent Behavior. Vol. 13, Issue 1 Jan.- Feb. 60-67.

3. Briones, Ma. Agnes C. (2007). Assertiveness, Hardiness and Coping Style in Relation to the Socio-demographic Profile of Abused adolescents: A Basis for a Proposed Rehabilitation Program. MA
Clinical Psychology University of Santo Tomas.

4. Buback, Debra III RN, 2004. Assertiveness Training to Prevent verbal Abuse in the Operating Room.AORN Vol 79, Issue 1 Jan. 147-164.

5. Butler, Pamela. Spring1992. Assertiveness Training: Teaching Women not to Discriminate against Themselves. Psychotherapy: Theory, Research and Practice Vol. 13 (1). 56-60.

6. Gallion, B.H., 1984. The effects of Instructional and Performance-based Assertiveness Training on Nonassertive and Anxious performer subjects. Dissertation Abstract International 44 (9B). 2892.

7. Glueckauf, Robert L., Quitness, Alexandra L., 1992. Assertiveness Training for Disabled.Adults in wheelchairs: Self-Report, Role-Play and Activity Pattern Outcomes. Journal of Consulting and Clinical Psychology. Vol. 60, Issue 3. June. 419-425.

8. Korsgard, M.A., Roberson L. \&Rymph R.D., 1998. What Motivates Fairness? The Role of Subordinate Assertive Behavior on Manager's Interactional Fairness. Journal of Applied Psychology.Vol 83.No. 5.731744.

9. Lange, A.J.; Jakubowski, P., 1976. Responsible assertive Behavior. Research Press, Champaign, IL.

10. Lardirzabal, M.G., 2001. Assessment of a Cooperative-Creative Program of Assertive Behavior and Self-Concept.Spanish Journal of Psychology. Vol. 2 No. 1.3-10.

11. Lee S., \&Crochett, M.S., 1994. Effect of Assertiveness Training on Levels of Stress and Assertiveness Experienced by Nurses in Taiwan, Republic of China. Issues in Mental Health Nursing.Vol 15 No.4.419-432.

12. Meyer, M.M., 1991. The Effects of Assertion Training on Assertive Behaviors, SelfAcceptance, Self-Concept and Locus of 
Control, Dissertation Abstract International 52 (4-B). 2331.

13. Morgan, Brenda and Leung, Paul. 1980. Effects of Assertion Training on Acceptance of Disability by Physically Abused University Students. Journal of Counseling Psychology. Vol.27, Issue 2.March.209-212

14. Myers, Mary B., Templer, Donald I., Brown, Ric. 1984. Coping Ability in Women who become Victims of Rape. Journal of Consulting and Clinical Psychology. Vol 52, Issue 1, February.73-78.

15. Pfost, K.S., Stevens, M.J., Parker, J.C. \& Mc Gowan, J.F., 1992. The Influence of Assertion Training on Three Aspects ofAssertiveness in Alcoholics. Journal of Clinical Psychology Vol. 48 No.12.262-268.

16. Santiago, Maureen V. (2004). The Adjustment Problems and Coping Strategies of First Year High School Students with Separated Parents: Basis for a Proposed Psychologicallntervention Program.MA Psychology.University Of Santo Tomas.

17. Sharon, R. A., 1997. Assertion Training Therapy in Psychiatric Milleus. Archives of Psychiatric Nursing.Vol 11 No. 1.46-51.

18. Weinhard, L.S., Carey, M.P., Carey, K.B. \&Verdecias, R.N., 1998. Increasing Assertiveness Skills to Reduce HIV Risk among Women with a Severe and Persistent Mental Illness. Journal of Consulting and Clinical Psychology.Vol 66 No. 4.660-684.

19. Weston\& Went, F., 1999. Speaking Up for Yourself: Description And Evaluation of Assertiveness Training Group for People with Learning disabilities, British Journal Disabilities, 27.110-115.

20. Wise K.L., Bundy, K.A., Bundy, E.A. \& Wise, L.A., 1991. Social Skills Training for Young Adolescents. Vol. 26.No. 101. 233-241. cited by Scopus.
21. Yen, Ru-Lin, I-Shin Shiah. Yu-Cune Chang et.al. 2004. Nurse Education Today. Vol. 24. Issue No. 4. 656-665.

\section{Other Materials}

1. A Collection of Pope John Paul II's Messages to the Youth, DSF 1994; Pasay City Philippines. A Booklet. 